\title{
FOURIER EXPANSIONS OF COMPLEX-VALUED EISENSTEIN SERIES ON FINITE UPPER HALF PLANES
}

\author{
ANTHONY SHAHEEN AND AUDREY TERRAS
}

Received 11 May 2006; Revised 20 July 2006; Accepted 25 July 2006

We consider complex-valued modular forms on finite upper half planes $H_{q}$ and obtain Fourier expansions of Eisenstein series invariant under the groups $\Gamma=\operatorname{SL}\left(2, \mathbb{F}_{p}\right)$ and $\mathrm{GL}\left(2, \mathbb{F}_{p}\right)$. The expansions are analogous to those of Maass wave forms on the ordinary Poincaré upper half plane - the $K$-Bessel functions being replaced by Kloosterman sums.

Copyright (c) 2006 Hindawi Publishing Corporation. All rights reserved.

\section{Introduction}

Before outlining our results, let us give a brief summary of the classical results for which we have found finite analogs. This work is a part of a continuing project to seek out finite analogs of Terras [17, Chapter 2].

The usual Poincare upper half plane $H$ consists of complex numbers $z=x+i y$ with $y>0$. The Poincaré arc length is defined by $d s^{2}=y^{-2}\left(d x^{2}+d y^{2}\right)$ and the corresponding Laplacian is $\Delta=y^{2}\left(\partial^{2} / \partial x^{2}+\partial^{2} / \partial y^{2}\right)$. More information about $H$, its non-Euclidean geometry, and modular forms can be found in [17].

Maass wave forms (of weight 0 ) for $\Gamma=\operatorname{SL}(2, \mathbb{Z})$ are functions $f: H \rightarrow \mathbb{C}$ which are eigenfunctions of the Poincaré Laplacian $\Delta=y^{2}\left(\partial^{2} / \partial x^{2}+\partial^{2} / \partial y^{2}\right)$ such that $f(\gamma z)=$ $f((a z+b) /(c z+d))=f(z)$ for all $z \in H$ and $\gamma=\left(\begin{array}{ll}a & b \\ c & d\end{array}\right) \in \Gamma$ and with $f(z)$ growing at most as a polynomial in $y$ as $y \rightarrow \infty$.

An example of a Maass wave form for $\mathrm{SL}(2, \mathbb{Z})$ is provided by the Maass Eisenstein series on $H$ defined, for $z \in H$ and $s \in \mathbb{C}$ with $\operatorname{Re} s>1$, by

$$
E_{s}(z)=\sum_{\gamma \in \Gamma_{\infty} \backslash \Gamma} \operatorname{Im}(\gamma z)^{s} .
$$

Here $\Gamma_{\infty}$ is the subgroup of $\Gamma$ consisting of matrices which fix $\infty$ (i.e., the lower left element is 0 ).

Define the gamma function for Res $>0$ by

$$
\Gamma(s)=\int_{0}^{\infty} y^{s} e^{-y} \frac{d y}{y} .
$$

Hindawi Publishing Corporation International Journal of Mathematics and Mathematical Sciences Volume 2006, Article ID 63918, Pages 1-17

DOI 10.1155/IJMMS/2006/63918 
2 Fourier expansions of Eisenstein series

Next define the $K$-Bessel function for $\operatorname{Re} a>0$ by

$$
K_{s}(a)=\frac{1}{2} \int_{0}^{\infty} y^{s} e^{-a / 2(y+(1 / y))} \frac{d y}{y} .
$$

Set

$$
\Lambda(s)=\pi^{-s} \Gamma(s) \zeta(2 s)
$$

where $\zeta(s)$ is Riemann's zeta function. Then the Fourier expansion of $E_{s}(z)$ is

$$
y^{s}+\frac{\Lambda(1-s)}{\Lambda(s)} y^{1-s}+\frac{2}{\Lambda(s)} \sum_{n \neq 0}|n|^{s-1 / 2} \sigma_{1-2 s}(n) y^{1 / 2} K_{s-1 / 2}(2 \pi|n| y) e^{2 \pi i n x} .
$$

Here the divisor function is

$$
\sigma_{s}(n)=\sum_{0<d \mid n} d^{s}
$$

See [17, pages 208-209] for a sketch of three proofs.

The upper half plane $H$ has a finite analog called the finite "upper" half plane $H_{q}$ attached to a finite field $\mathbb{F}_{q}$ with $q$ elements. We assume $q$ is an odd number. It is likely that most of our results go through in characteristic 2, but we leave this for a future paper. Then we take a fixed nonsquare $\delta \in \mathbb{F}_{q}$ and define

$$
H_{q}=\left\{z=x+y \sqrt{\delta} \mid x, y \in \mathbb{F}_{q}, y \neq 0\right\} .
$$

The finite upper half plane is considered in detail in [18, Chapter 19]. See also [13]. An application to coding theory is to be found in [19]. References which cover finite upper half planes in both even and odd characteristics include Angel [1], Angel and Evans [3], Evans [8], as well as Soto-Andrade and Vargas [15].

Recall that an element of the general linear group

$$
g=\left(\begin{array}{ll}
a & b \\
c & d
\end{array}\right) \in \mathrm{GL}\left(2, \mathbb{F}_{q}\right)
$$

has entries in $\mathbb{F}_{q}$ and nonzero determinant. Then for $z \in H_{q}$,

$$
g z=\frac{a z+b}{c z+d} \in H_{q}
$$

Define the "distance" between $z, w \in H_{q}$ by

$$
d(z, w)=\frac{N(z-w)}{\operatorname{Im} z \operatorname{Im} w} .
$$

It is easily checked that for $g \in \mathrm{GL}\left(2, \mathbb{F}_{q}\right), d(g w, g w)=d(z, w)$. For $a \in \mathbb{F}_{q}$, define the finite upper half plane graphs $X_{q}(\delta, a)$ to have vertices the elements of $H_{q}$ and an edge between $z$ and $w$ in $H_{q}$ if and only if $d(z, w)=a$. For $a \neq 0,4 \delta$, the graph $X_{q}(\delta, a)$ is $(q+1)$-regular, 
connected, and Ramanujan. See [18] for more information. Ramanujan graphs are of interest to computer scientists because they provide efficient communications networks. Chai and $\mathrm{Li}$ [7] have proved some interesting connections of the spectra of the finite upper half plane graphs and modular forms of function fields.

The GL(2, $\left.\mathbb{F}_{q}\right)$-invariant operators on $H_{q}$ analogous to the non-Euclidean Laplacian on the Poincaré upper half plane $H$ are the adjacency operators on the finite upper half plane graphs defined for $a \in \mathbb{F}_{q}$ by

$$
A_{a} f(z)=\sum_{\substack{w \in H_{q} \\ d(z, w)=a}} f(w) .
$$

These operators generate a commutative algebra of operators.

One can view $H_{q}$ as a finite symmetric space $G / K$ as in [18, Chapter 19]. From that point of view, the adjacency operators $A_{a}$ correspond to $G$-invariant differential operators on a symmetric space. This subject may also be studied from the point of view of association schemes (Bannai and Ito [4]) or Hecke operators (Krieg [11]).

A (complex-valued) modular form on the finite upper half plane $H_{q}$ is a function $f$ : $H_{q} \rightarrow \mathbb{C}$ having an invariance property for a subgroup $\Gamma$ of $\mathrm{GL}\left(2, \mathbb{F}_{q}\right)$. See Definition 2.4. Here we consider $\Gamma=\operatorname{SL}\left(2, \mathbb{F}_{p}\right)$ and $\mathrm{GL}\left(2, \mathbb{F}_{p}\right)$. Such modular forms which are eigenfunctions of the adjacency operators for the finite upper half plane graphs attached to $H_{q}$ are analogs of Maass wave forms on the Poincaré upper half plane.

Now we consider one of our finite upper half plane analogs of the Maass Eisenstein series in formula (1.1). Let $\Gamma$ be a subgroup of $\mathrm{GL}\left(2, \mathbb{F}_{q}\right)$ and let $\chi$ be a multiplicative character on $\mathbb{F}_{q}^{*}$. Define the Eisenstein "series" for $z \in H_{q}$ as

$$
E_{\chi, \Gamma}(z)=\sum_{\gamma \in \Gamma} \chi(\operatorname{Im}(\gamma z))
$$

We will see that $E_{\chi, \Gamma}$ is an eigenfunction of the adjacency operators of finite upper half plane graphs if it does not vanish identically on $H_{q}$. To see when this happens, recall that $\mathbb{F}_{q}^{*}$ is a cyclic group with generator $g$. (See [18] and the references given there for more information about finite fields.) Thus a multiplicative character $\chi$ of $\mathbb{F}_{q}^{*}$ has the following form for integers $a, b$ :

$$
\chi_{a}\left(g^{b}\right)=e^{2 \pi i a b /(q-1)}, \quad 0 \leq a, b \leq q-2 .
$$

We will find that for $q=p^{n}$, with $q>2$ and $n>2, E_{\chi, \mathrm{GL}\left(2, \mathbb{F}_{q}\right)}$ is nonzero if and only if $\chi=\chi_{a}$ when $a$ is a multiple of $(p-1)$. This last condition is equivalent to saying that $\left.\chi_{a}\right|_{\mathbb{F}_{p}^{*}}=1$. When $n=2$, we will find that $E_{\lambda, \mathrm{GL}\left(2, \mathbb{F}_{q}\right)}$ is nonzero, for $\chi=1$ or $\chi=\lambda$ as in formula (1.20). We will also find experimentally that $E_{\chi, \mathrm{GL}\left(2, \mathbb{F}_{q}\right)}$ is nonzero for $p=3,5$ when $a$ is a multiple of $(p-1)$ (see Tables 2.1 and 2.2 near the end of the paper).

As we saw in formula (1.5), Fourier expansions of Maass Eisenstein series on $H$ involve $K$-Bessel functions. In the finite case under consideration, the Bessel functions will be replaced with Kloosterman sums. Thus we need to define these sums as well as Gauss sums in order to state the Fourier expansion of the Eisenstein series $E_{\chi, \mathrm{GL}\left(2, \mathbb{F}_{p}\right)}(z)$. 
4 Fourier expansions of Eisenstein series

Let $\Psi$ be an additive character of $\mathbb{F}_{q}$. Then $\Psi=\Psi_{b}$, where

$$
\Psi_{b}(u)=e^{2 \pi i \operatorname{Tr}(b u) / p}, \quad \text { for } b, u \in \mathbb{F}_{q} .
$$

Here the trace in the exponent is (for $q=p^{n}, p=$ prime)

$$
\operatorname{Tr}(u)=\operatorname{Tr}_{\mathbb{F}_{q} / \mathbb{F}_{p}}(u)=u+u^{p}+u^{p^{2}}+\cdots+u^{p^{n-1}} .
$$

Let $\Psi$ be an additive character and $\chi$ a multiplicative character. Define the Gauss sum by

$$
\Gamma(\chi, \Psi)=\Gamma_{q}(\chi, \Psi)=\sum_{t \in \mathbb{F}_{q}^{*}} \chi(t) \Psi(t)
$$

The Gauss sum is analogous to the gamma function of formula (1.2).

See [10] by Ireland and Rosen for more information on Gauss sums. In particular, they show that if $\chi$ and $\Psi_{b}$ are not trivial, then

$$
\Gamma\left(\chi, \Psi_{b}\right)=\chi\left(b^{-1}\right) \Gamma\left(\chi, \Psi_{1}\right) .
$$

Another basic result says that if $\chi$ and $\Psi$ are not trivial, then

$$
\left|\Gamma_{q}(\chi, \Psi)\right|=\sqrt{q} .
$$

The Kloosterman sum for $a, b \in \mathbb{F}_{q}$ is defined by

$$
K_{\Psi}(\chi \mid a, b)=\sum_{t \in \mathbb{F}_{q}^{*}} \chi(t) \Psi\left(a t+b t^{-1}\right) .
$$

The Kloosterman sum is analogous to the $K$-Bessel function of formula (1.3). These sums have great importance in number theory. See [12] by Sarnak who discusses the connection with modular forms and "Kloostermania."

Define the quadratic residue character $\lambda$ on $\mathbb{F}_{q}^{*}$ by

$$
\lambda(y)= \begin{cases}1, & \text { if } y \text { is a square in } \mathbb{F}_{q}^{*}, \\ -1, & \text { if } y \text { is not a square in } \mathbb{F}_{q}^{*}, \\ 0, & y=0 .\end{cases}
$$

One of our main results (see formula (2.34)) is a finite analog of formula (1.5) and says that the Fourier expansion of the Eisenstein series $E_{\chi, \mathrm{GL}\left(2, \mathbb{F}_{q}\right)}(z)$ is given by

$$
\begin{aligned}
\frac{1}{p(p-1)^{2}} E_{\chi, \mathrm{GL}\left(2, \mathbb{F}_{q}\right)}(z)= & \chi(y)+\frac{p}{q} \frac{\Gamma\left(\lambda, \Psi_{1}\right) \Gamma\left(\lambda \chi, \Psi_{1}\right)}{\Gamma\left(\chi, \Psi_{1}\right)} \chi^{-1}(-\delta y) \lambda(-\delta) \\
& +\frac{p}{q} \frac{\Gamma\left(\lambda, \Psi_{1}\right)}{\Gamma\left(\chi, \Psi_{1}\right)} \chi(y) \sum_{\substack{b \in \mathbb{F}_{q}^{*} \\
\operatorname{Tr}(b)=0}} \frac{\chi(b)}{\lambda(b)} K_{\Psi_{b}}\left(\lambda \chi \mid-\delta y^{2},-\frac{1}{4}\right) \Psi_{b}(-x) .
\end{aligned}
$$


We will also prove the analogous result for $\Gamma=\operatorname{SL}\left(2, \mathbb{F}_{p}\right)$ in formula (2.32). Both of these Fourier expansions result from that of Theorem 2.9 which gives the Fourier expansion of what we call a $G$-type Eisenstein series (see Definition 2.2).

The main idea of the proof of (1.21) is to imitate the second proof of formula (1.5) given by Terras [17]. This proof goes back to Bateman and Grosswald [5] and is extended by Terras [16]. The main fact needed is the Poisson summation formula for $\mathbb{Z}$ as a subgroup of $\mathbb{R}$. For our finite upper half plane analog we will use Poisson summation for the additive subgroup $\mathbb{F}_{p}$ of $\mathbb{F}_{q}$. See Proposition 2.5 or [18, page 199].

One can also consider finite field valued modular forms on finite upper half planes. These are perhaps more analogous to holomorphic modular forms. See [13] by Shaheen where an analog of the Selberg trace formula is developed which is more like that of Selberg (Hejhal [9]) than the version of Terras [18].

\section{Lattices, modular forms on $H_{q}$, and Fourier expansions}

Classically Eisenstein series are sums over lattices such as $\mathbb{Z} w_{1} \oplus \mathbb{Z} w_{2}$ for elements $w_{i}$ in $\mathbb{C}$ which are linearly independent over $\mathbb{R}$. Our finite analog of a lattice is defined as follows. Here $q=p^{n}$, with $n>1$.

Definition 2.1. An $\mathbb{F}_{p}$-lattice in $\mathbb{C}_{q}=\mathbb{F}_{q}(\sqrt{\delta})$ is a set of the form $L=\mathbb{F}_{p} w_{1} \oplus \mathbb{F}_{p} w_{2}$, where $w_{1}, w_{2} \in \mathbb{C}_{q}$ are linearly independent over $\mathbb{F}_{q}$.

Note that if $z \in H_{q}$, we have an example of an $\mathbb{F}_{p}$-lattice in $\mathbb{C}_{q}=\mathbb{F}_{q}(\sqrt{\delta})$ by setting $L_{z}=\mathbb{F}_{p} z \oplus \mathbb{F}_{p}$. Next we define a $G$-type Eisenstein series.

Definition 2.2. Let $\chi: \mathbb{F}_{q}(\sqrt{\delta})^{*} \rightarrow \mathbb{C}$ be a multiplicative character. Define the G-Eisenstein series $G_{\chi}(z)$, for $z \in H_{q}$, by

$$
G_{\chi}(z)=\sum_{\substack{0 \neq \omega \\ \omega \in L_{z}=\mathbb{F}_{p} z \oplus \mathbb{F}_{p}}} \chi\left(\frac{1}{\omega}\right)
$$

Proposition 2.3. If $q=p^{n}, \gamma=\left(\begin{array}{ll}a & b \\ c & d\end{array}\right) \in \mathrm{GL}\left(2, \mathbb{F}_{p}\right)$, and $z \in H_{q}$, then

$$
G_{\chi}(\gamma z)=\chi(c z+d) G_{\chi}(z)
$$

Proof. Imitate the classical proof found in [14].

We now give our most general definition of a complex-valued modular form.

Definition 2.4. Let $\chi: \mathbb{F}_{q}(\sqrt{\delta})^{*} \rightarrow \mathbb{C}$ be a multiplicative character. Define $f: H_{q} \rightarrow \mathbb{C}$ to be a complex-valued modular form for $\chi$ and a subgroup $\Gamma$ of $\mathrm{GL}\left(2, \mathbb{F}_{q}\right)$ if

$$
f(\gamma z)=\chi(c z+d) f(z) \quad \forall \gamma=\left(\begin{array}{ll}
a & b \\
c & d
\end{array}\right) \in \Gamma, z \in H_{q} .
$$

We denote the complex vector space of such forms by $M_{\chi}(\Gamma)$.

Proposition 2.3 can then be restated to say that, as a function of $z, G_{\chi}(z) \in M_{\chi}(\Gamma)$. If $f \in M_{\chi}(\Gamma)$ and $g \in M_{\psi}(\Gamma)$, then $f g \in M_{\chi \psi}(\Gamma)$. 


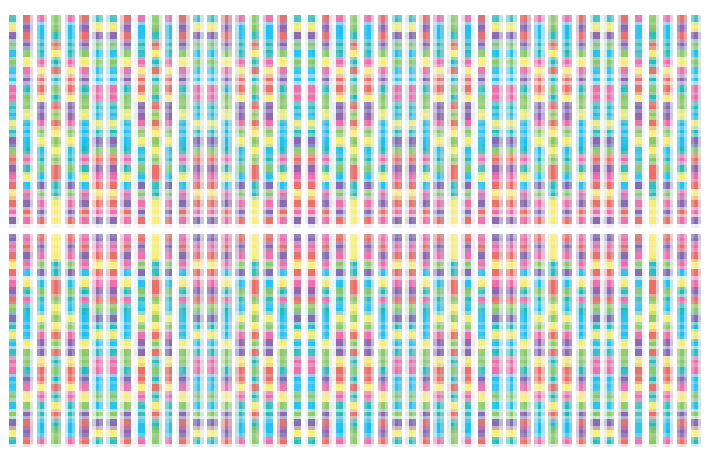

Figure 2.1. A tessellation of $H_{49}$ from $\mathrm{GL}\left(2, \mathbb{F}_{7}\right)$. Any 7 points of different colors form a fundamental domain for $\operatorname{GL}\left(2, \mathbb{F}_{7}\right) \backslash H_{49}$.

Note that $M_{\chi}\left(\mathrm{GL}\left(2, \mathbb{F}_{p}\right)\right) \neq\{0\}$ implies that $\chi(a)=1$ for all $a \in \mathbb{F}_{p}^{*}$. Similarly,

$$
M_{\chi}\left(\operatorname{SL}\left(2, \mathbb{F}_{p}\right)\right) \neq\{0\}
$$

implies that $\chi(-1)=1$. This is analogous to the fact that classical holomorphic modular forms for $\operatorname{SL}(2, \mathbb{Z})$ must have even weight.

Fundamental domains for $\Gamma \backslash H_{q}$ are discussed by Shaheen [13]. It turns out that the classical perpendicular bisector method for constructing such fundamental domains (discussed by Terras [17], e.g.) has a finite analog.

Tessellations of $H_{q}$ given by letting $\Gamma$ act on a fundamental domain are as beautiful as those for the modular group acting on the Poincaré upper half plane. One such tessellation is given in Figure 2.1.

Let $\chi$ be a multiplicative character of $\mathbb{F}_{q}^{*}$ and let $N=N_{\mathbb{F}_{q}(\sqrt{\delta}) / \mathbb{F}_{q}}$ be the norm map. The character $\chi \circ N^{-1}$ seems like a reasonable character to investigate. In fact, it comes up when we consider the Eisenstein series $E_{\chi, \Gamma}$ from formula (1.12) of the introduction when $\Gamma=\mathrm{GL}\left(2, \mathbb{F}_{p}\right)$ as we will soon see.

Here we will need the Poisson summation formula for a subgroup $B$ of a finite abelian group $A$ proved by Terras [18, page 199]. The formula relates Fourier analysis on $A / B$ and that on $A$ itself.

Proposition 2.5 (Poisson summation formula for finite abelian groups). Suppose $B$ is a subgroup of the finite additive abelian group $A$ and $f: A \rightarrow \mathbb{C}$. Then

$$
\frac{1}{|B|} \sum_{b \in B} f(a+b)=\frac{1}{|A|} \sum_{\Psi \in B^{\sharp}} \hat{f}(\Psi) \Psi(a) .
$$

Write $\hat{A}$ to denote the group of additive characters of $A$. Here the dual group to $B$ in $A$ is

$$
B^{\#}=\{\Psi \in \widehat{A} \mid \Psi(b)=1, \forall b \in B\} \cong \widehat{(A / B)}
$$


and the Fourier transform of $f$ at $\Psi \in \widehat{A}$ is

$$
\hat{f}(\Psi)=\sum_{u \in A} f(u) \bar{\Psi}(u)
$$

Note that the left-hand side of formula (2.5) is a function of the coset $a+B$ in $A / B$ while the right-hand side involves Fourier transforms on $A$.

We want to apply the Poisson summation formula to $B=\mathbb{F}_{p}$ as an additive subgroup of $A=\mathbb{F}_{q}$. Therefore, we will need to know the characters in $\mathbb{F}_{p}^{\#}$. As in formula (1.14), $\Psi_{a}(u)=e^{2 \pi i \operatorname{Tr}(a u) / p}$ is an arbitrary element of $\widehat{\mathbb{F}}_{q}$. Here the trace is $\operatorname{Tr}=\operatorname{Tr}_{\mathbb{F}_{q} / \mathbb{F}_{p}}$ as in formula (1.15). Then, $\Psi_{a}(u)=e^{2 \pi i \operatorname{Tr}(a u) / p}=1$ for all $u \in \mathbb{F}_{p}$ if and only if $p$ divides $\operatorname{Tr}(a u)=$ $u \operatorname{Tr}(a)$ for all $u \in \mathbb{F}_{p}$. Therefore, $\Psi_{a} \in \mathbb{F}_{p}^{\#}$ if and only if $\operatorname{Tr}(a)=0$ in $\mathbb{F}_{p}$. We have proved the following lemma.

LEMMA 2.6. Using the notation of the preceding proposition, if $\mathbb{F}_{p}$ is considered as a subgroup of the additive group $\mathbb{F}_{q}$, the dual group to $\mathbb{F}_{p}$ in $\mathbb{F}_{q}$ is

$$
\mathbb{F}_{p}^{\#}=\left\{\Psi_{a} \in \widehat{\mathbb{F}}_{q} \mid a \in \mathbb{F}_{q}, \operatorname{Tr}(a)=\operatorname{Tr}_{\mathbb{F}_{q} / \mathbb{F}_{p}}(a)=0\right\} .
$$

We will also find that the Kloosterman sum appears in a different guise in our computations. So let us define the finite $k$-Bessel function in analogy to that considered on the Poincaré upper half plane in [17, page 136].

Definition 2.7. For a multiplicative character $\chi$ of $\mathbb{F}_{q}^{*}$ and an additive character $\Psi$ of $\mathbb{F}_{q}$, if $z \in H_{q}$, define the $k$-Bessel function by

$$
k(z \mid \chi, \Psi)=\sum_{u \in \mathbb{F}_{q}} \chi\left(\operatorname{Im}\left(\frac{-1}{z+u}\right)\right) \Psi(u) .
$$

It follows from Lemma 2.11 that $k(z)=k(z \mid \chi, \Psi)$ is an eigenfunction of all the adjacency operators $A_{a}$ for the finite upper half plane graphs from formula (1.11).

The following proposition is proved by Celniker et al. [6] (see also [2]). It is a finite analog of a classical integral formula for $K$-Bessel functions.

Proposition 2.8 (Relation between $k$-Bessel function and Kloosterman sum). For a nontrivial multiplicative character $\chi$ of $\mathbb{F}_{q}^{*}$ and a nontrivial additive character $\Psi_{a}$ of $\mathbb{F}_{q}$ and $z \in H_{q}$,

$$
\Gamma\left(\chi, \Psi_{a}\right) k\left(z \mid \chi, \Psi_{a}\right)=g_{a} \chi(y) \Psi_{a}(-x) K_{\Psi_{a}}\left(\chi \lambda \mid-\delta y^{2},-\frac{1}{4}\right)
$$

where $g_{a}=\sum_{u \in \mathbb{F}_{q}} \Psi_{a}\left(u^{2}\right)$ is a Gauss sum, the Kloosterman sum $K$ is defined by formula (1.19), and the multiplicative character $\lambda$ is given in formula (1.20).

Why do we say that $g_{a}$ is a Gauss sum? It is easily seen that for $a \neq 0$,

$$
g_{a}=\sum_{u \in \mathbb{F}_{q}} \Psi_{a}\left(u^{2}\right)=\sum_{u \in \mathbb{F}_{q}}(1+\lambda(u)) \Psi_{a}(u)=\lambda\left(a^{-1}\right) \Gamma\left(\lambda, \Psi_{1}\right) .
$$


Theorem 2.9. Let $N: \mathbb{F}_{q}(\sqrt{\delta})^{*} \rightarrow \mathbb{F}_{q}^{*}$ be the norm map, $\chi=\chi_{a}$ the multiplicative character of $\mathbb{F}_{q}^{*}$ given by (1.13), and $z \in H_{q}$. If $a=0$, then $G_{\chi_{0}}(z)=p^{2}-1$. If

$$
a \in\left\{\frac{p-1}{2}, \frac{2(p-1)}{2}, \frac{3(p-1)}{2}, \frac{4(p-1)}{2}, \ldots, \frac{m(p-1)}{2}\right\},
$$

where $m / 2=(q-1) /(p-1)-1$, then for $z=x+y \sqrt{\delta} \in H_{q}$, the G-Eisenstein series has the following Fourier expansion:

$$
\begin{aligned}
\frac{1}{p-1} G_{\chi \circ N^{-1}}(z)= & 1+\frac{p}{q} \frac{\Gamma\left(\lambda, \Psi_{1}\right) \Gamma\left(\lambda \chi, \Psi_{1}\right)}{\Gamma\left(\chi, \Psi_{1}\right)} \chi^{-1}\left(-\delta y^{2}\right) \lambda^{-1}(-\delta) \\
& +\frac{p}{q} \frac{\Gamma\left(\lambda, \Psi_{1}\right)}{\Gamma\left(\chi, \Psi_{1}\right)} \sum_{\substack{0 \neq b \in \mathbb{F}_{q} \\
\operatorname{Tr}(b)=0}}(\chi \lambda)(b) K_{\Psi_{b}}\left(\lambda \chi \mid-\delta y^{2},-\frac{1}{4}\right) \psi_{b}(-x) .
\end{aligned}
$$

Here $\psi_{b}$ is the additive character of $\mathbb{F}_{q}$ given by $(1.14), \Gamma(\chi, \Psi)$ is the Gauss sum from formula (1.16), $K_{\psi}$ is the Kloosterman sum from formula (1.19), and $\lambda$ is the multiplicative character from formula (1.20). If

$$
a \notin\left\{0, \frac{p-1}{2}, \frac{2(p-1)}{2}, \frac{3(p-1)}{2}, \frac{4(p-1)}{2}, \ldots, \frac{m(p-1)}{2}\right\}
$$

then $G_{\chi \circ N^{-1}}=0$.

Proof. First note that $a \in\{0,(p-1) / 2,2(p-1) / 2,3(p-1) / 2,4(p-1) / 2, \ldots, m(p-1) / 2\}$ is equivalent to $\chi_{a}^{2}=i d e n t i t y$ on $\mathbb{F}_{p}^{*}$. To see this, suppose that $\mathbb{F}_{q}^{*}=\langle g\rangle$ and $\chi=\chi_{a}$ as in formula (1.13). It follows that $\mathbb{F}_{p}^{*}=\left\langle g^{(q-1) /(p-1)}\right\rangle$. Therefore, $\chi^{2}=$ identity on $\mathbb{F}_{p}^{*}$ if and only if $(p-1)$ divides $2 a$.

Henceforth, we will assume that $\chi^{2}=$ identity on $\mathbb{F}_{p}^{*}$. The case $\chi=\chi_{0}$ is trivial since $G_{\chi}$ is constant. Thus for the remainder of this proof we also assume that $\chi \neq \chi_{0}$.

If $z \in H_{q}$, then

$$
\begin{aligned}
G_{\chi \circ N^{-1}}(z) & =\sum_{\substack{(c, d) \in \mathbb{F}_{p}^{2} \\
(c, d) \neq(0,0)}} \chi\left(\frac{1}{N(c z+d)}\right)=\sum_{d \in \mathbb{F}_{p}^{*}} \chi\left(\frac{1}{d^{2}}\right)+\sum_{c \in \mathbb{F}_{p}^{*}} \chi\left(\frac{1}{c^{2}}\right) \sum_{d \in \mathbb{F}_{p}} \chi^{-1}\left(N\left(z+\frac{d}{c}\right)\right) \\
& =\sum_{v \in \mathbb{F}_{p}^{*}} \chi^{2}(v)+\sum_{v \in \mathbb{F}_{p}^{*}} \chi^{2}(v) \sum_{u \in \mathbb{F}_{p}} \chi^{-1}(N(z+u)) \\
& =(p-1) \begin{cases}0, & \text { if } \chi^{2} \neq \text { identity on } \mathbb{F}_{p}^{*}, \\
1+\sum_{u \in \mathbb{F}_{p}} \chi^{-1}(N(z+u)), & \text { if } \chi^{2}=\text { identity on } \mathbb{F}_{p}^{*} .\end{cases}
\end{aligned}
$$


We can now apply Poisson summation (from Proposition 2.5, (2.5)) and Lemma 2.6 to see that

$$
\sum_{u \in \mathbb{F}_{p}} \chi^{-1}(N(z+u))=\frac{p}{q} \sum_{\substack{0 \neq b \in \mathbb{F}_{q} \\ \operatorname{Tr}(b)=0}} \sum_{u \in \mathbb{F}_{q}} \chi^{-1}(N(z+u)) \overline{\Psi_{b}}(u)
$$

Expanding the inner sum, we have, using Definition 2.7, for $b \neq 0$,

$$
\begin{aligned}
\sum_{u \in \mathbb{F}_{q}} \chi^{-1}(N(z+u)) \Psi_{-b}(u) & =\sum_{u \in \mathbb{F}_{q}} \chi\left(\frac{y(1 / y)}{N(z+u)}\right) \Psi_{-b}(u)=\chi^{-1}(y) k\left(z \mid \chi, \Psi_{-b}\right) \\
& =\frac{g_{-b}}{\Gamma\left(\chi, \Psi_{-b}\right)} \Psi_{-b}(-x) K_{\Psi_{-b}}\left(\chi \lambda \mid-\delta y^{2},-\frac{1}{4}\right) .
\end{aligned}
$$

Here we make use of Proposition 2.8.

It follows from formula (2.11) that

$$
\begin{aligned}
\frac{1}{p-1} G_{\chi \circ N^{-1}}(z)= & 1+\frac{p}{q \chi(y)} k\left(z \mid \chi, \Psi_{0}\right) \\
& +\frac{p}{q} \sum_{\substack{0 \neq b \in \mathbb{F}_{q} \\
\operatorname{Tr}(b)=0}} \frac{\Gamma\left(\lambda, \Psi_{1}\right)}{\Gamma\left(\chi, \Psi_{b}\right)} \lambda\left(b^{-1}\right) K_{\Psi_{b}}\left(\chi \lambda \mid-\delta y^{2},-\frac{1}{4}\right) \psi_{b}(-x) .
\end{aligned}
$$

Apply formula (1.17) to see that

$$
\begin{aligned}
\frac{1}{p-1} G_{\chi \circ N^{-1}}(z)= & 1+\frac{p}{q \chi(y)} k\left(z \mid \chi, \Psi_{0}\right) \\
& +\frac{p}{q} \frac{\Gamma\left(\lambda, \Psi_{1}\right)}{\Gamma\left(\chi, \Psi_{1}\right)} \sum_{\substack{0 \neq b \in \mathbb{E}_{q} \\
\operatorname{Tr}(b)=0}}(\chi \lambda)(b) K_{\Psi_{b}}\left(\chi \lambda \mid-\delta y^{2},-\frac{1}{4}\right) \psi_{b}(-x) .
\end{aligned}
$$

But, what is $k\left(z \mid \chi, \Psi_{0}\right)$ ? To answer this, note that

$$
k\left(z \mid \chi, \Psi_{0}\right)=\sum_{u \in \mathbb{F}_{q}} \chi\left(\operatorname{Im}\left(\frac{-1}{z+u}\right)\right)=\frac{1}{\chi(y)} \sum_{v \in \mathbb{F}_{q}} \chi^{-1}\left(v^{2}-\delta\right) .
$$

Plug in formula (1.17),

$$
\chi^{-1}\left(v^{2}-\delta\right)=\frac{\Gamma\left(\chi, \Psi_{v^{2}-\delta}\right)}{\Gamma\left(\chi, \Psi_{1}\right)}
$$


to obtain

$$
\begin{aligned}
k\left(z \mid \chi, \Psi_{0}\right) & =\frac{1}{\chi(y)} \sum_{v \in \mathbb{F}_{q}} \frac{\Gamma\left(\chi, \Psi_{v^{2}-\delta}\right)}{\Gamma\left(\chi, \Psi_{1}\right)}=\frac{1}{\chi(y) \Gamma\left(\chi, \Psi_{1}\right)} \sum_{v \in \mathbb{F}_{q}} \sum_{w \in \mathbb{F}_{q}^{*}} \chi(w) e^{2 \pi i \operatorname{Tr}\left(w\left(v^{2}-\delta\right)\right) / p} \\
& =\frac{1}{\chi(y) \Gamma\left(\chi, \Psi_{1}\right)} \sum_{w \in \mathbb{F}_{q}^{*}} \chi(w) e^{2 \pi i \operatorname{Tr}(-\delta w) / p} \sum_{v \in \mathbb{F}_{q}} e^{2 \pi i \operatorname{Tr}\left(w v^{2}\right) / p} .
\end{aligned}
$$

When $w \neq 0$, the inner sum is the Gauss sum $g_{w}=\lambda\left(w^{-1}\right) \Gamma\left(\lambda, \Psi_{1}\right)$. Therefore,

$$
k\left(z \mid \chi, \Psi_{0}\right)=\frac{\Gamma\left(\lambda, \Psi_{1}\right)}{\chi(y) \Gamma\left(\chi, \Psi_{1}\right)} \sum_{w \in \mathbb{F}_{q}^{*}}(\lambda \chi)(w) e^{2 \pi i \operatorname{Tr}(-\delta w) / p}=\frac{\Gamma\left(\lambda, \Psi_{1}\right) \Gamma\left(\lambda \chi, \Psi_{1}\right)}{\chi(-\delta y) \lambda(-\delta) \Gamma\left(\chi, \Psi_{1}\right)} .
$$

The theorem follows.

Furthermore, if $q \neq p^{2}$, then one can show that $G_{\chi \circ N^{-1}}(z)$ is not the zero function by showing that the constant term of its Fourier expansion is nonzero. When $q=p^{2}$, our later computations show that certain Eisenstein series must be nonzero also. But we leave the general $q=p^{2}$ question open for the moment.

Corollary 2.10. If $q \neq p^{2}$ and

$$
a \in\left\{0, \frac{p-1}{2}, \frac{2(p-1)}{2}, \frac{3(p-1)}{2}, \frac{4(p-1)}{2}, \ldots, \frac{m(p-1)}{2}\right\},
$$

where $m / 2=(q-1) /(p-1)-1$, then $G_{\chi_{a} \circ N^{-1}}(z)$ is not the zero function. If $q=p^{2}, G_{\lambda \circ N^{-1}}(z)$ is not the zero function.

Proof. If $a=0$, then the result is clear. If $a \neq 0$, we show that the constant term of the Fourier expansion of $G_{\chi_{a} \circ N^{-1}}(z)$ is nonzero. We have two cases to consider.

If $\lambda_{\chi_{a}}$ is the identity character, then the constant term of $(1 /(p-1)) G_{\chi_{a} \circ N^{-1}}(z)$ is

$$
\left(1+\frac{p}{q}\right) \Gamma\left(1, \Psi_{1}\right)=1-\frac{p}{q} \neq 0
$$

Suppose that $\lambda \chi_{a}$ is not the identity character. We take the absolute value of the second term in the constant term and note that it is not 1 using formula (1.18). Therefore, the constant term of $G_{\chi_{a} \circ N^{-1}}(z)$ is nonzero.

Next we consider the Fourier expansion of some Eisenstein series which are analogous to the Maass Eisenstein series (1.1). First, we define the finite analog of the power function $y^{s}$ on the Poincaré upper half plane.

Let $\chi: \mathbb{F}_{q}^{*} \rightarrow \mathbb{C}$ be a multiplicative character. Define the "power function" $p_{\chi}: H_{q} \rightarrow \mathbb{C}$ to be

$$
p_{\chi}(z)=\chi(\operatorname{Im}(z))
$$


Lemma 2.11 (The power function is an eigenfunction of all adjacency operators). If $A_{a}$ denotes the adjacency operator defined by formula (1.11), then

$$
A_{a} p_{\chi}=\mu(a, \chi) p_{\chi}, \quad \text { where } \mu(a, \chi)=\sum_{d(w, \sqrt{\delta})=a} \chi(\operatorname{Im}(w)) .
$$

Proof. See [18, page 324] by Terras.

Now we want to relate the Eisenstein series of E-type from formula (1.12) - an Eisenstein series analogous to the Maass Eisenstein series of formula (1.1) - and the G-type Eisenstein series from Definition 2.2. If $\Gamma$ is a subgroup of $\mathrm{GL}\left(2, \mathbb{F}_{q}\right), \chi$ is a multiplicative character on $\mathbb{F}_{q}^{*}$, and $p_{\chi}(z)$ is the power function from formula (2.26), define the finite upper half plane $E$-type Eisenstein series $E_{\chi, \Gamma}(z)$ by formula (1.12). Then we see that

$$
E_{\chi, \Gamma}(z)=\sum_{\gamma \in \Gamma} p_{\chi}(\gamma z)=\sum_{\gamma=\left(\begin{array}{l}
a \\
c \\
c
\end{array}\right) \in \Gamma} \chi\left(\frac{\operatorname{det}(\gamma) \operatorname{Im}(z)}{N(c z+d)}\right) .
$$

Note that $E_{\chi, \Gamma} \in M_{\chi_{1}}(\Gamma)$, where $\chi_{1}$ denotes the trivial character.

Lemma 2.12. Given $c, d \in \mathbb{F}_{p}$ not both zero and $u \in \mathbb{F}_{p}^{*}$, there exist $p$ pairs $(a, b) \in \mathbb{F}_{p}^{2}$ such that $\operatorname{det}\left(\begin{array}{ll}a & b \\ c & d\end{array}\right)=u$.

Proof. We need to solve the equation $x d-y c=u \neq 0$ for $x, y \in \mathbb{F}_{p}$. If $c \neq 0$, then we can pick any $x \in \mathbb{F}_{p}$ and solve for $y=c^{-1}(x d-u)$. This gives us $p$ different choices for $x$. If $c=0$, then $y$ can be any element of $\mathbb{F}_{p}$ and $x=u d^{-1}$. Again, we have $p$ choices for $y$.

We see from the preceding results that for our two favorite subgroups of GL(2, $\left.\mathbb{F}_{q}\right)$, we have the following relations between the E- and G-type Eisenstein series:

$$
\begin{gathered}
E_{\chi, \mathrm{SL}\left(2, \mathbb{F}_{p}\right)}(z)=p \chi(\operatorname{Im}(z)) G_{\chi \circ N^{-1}}(z), \\
E_{\chi, \mathrm{GL}\left(2, \mathbb{F}_{p}\right)}(z)=p(p-1) \chi(\operatorname{Im}(z)) G_{\chi \circ N^{-1}}(z) .
\end{gathered}
$$

Here the $G$-type Eisenstein series $G_{\chi}$ is defined by formula (2.1). In both cases of formula (2.29), when $\left.\chi^{2}\right|_{\mathbb{F}_{p}^{*}} \neq 1$, the Eisenstein series is 0 .

It follows from Lemma 2.11 that if $E_{\chi, \Gamma} \not \equiv 0$ on $H_{q}$, then $E_{\chi, \Gamma}(z)$ is an eigenfunction of the adjacency operator $A_{a}$ of formula (1.11) with eigenvalue

$$
\mu(a, \chi)=\sum_{\substack{w \in H_{q} \\ d(w, \sqrt{\delta})=a}} \chi(\operatorname{Im}(w)) .
$$

Thus the Eisenstein series, $E_{\chi, \Gamma}$, are the analogs of the classical Maass Eisenstein series $E_{s}$ from formula (1.1) at least when they are not identically zero. Angel et al. [2] considered Eisenstein series for $\Gamma=K$, the subgroup of $\mathrm{GL}\left(2, F_{q}\right)$ fixing $\sqrt{\delta}$. In this case, the Eisenstein series are usually called spherical functions. The general case was introduced in [13] in an attempt to find elements of $L^{2}\left(\Gamma \backslash H_{q}\right)$ that simultaneously diagonalize the matrices $A_{a}^{\Gamma}=\left.A_{a}\right|_{L^{2}\left(\Gamma \backslash H_{q}\right)}$. 
We can now easily find the Fourier expansions of $E_{\chi, \operatorname{SL}\left(2, \mathbb{F}_{p}\right)}$ and $E_{\chi, \mathrm{GL}\left(2, \mathbb{F}_{p}\right)}$ using that of $G_{\chi \circ N^{-1}}(z)$ in Theorem 2.9.

Let $\chi=\chi_{a}$ be the multiplicative character of $\mathbb{F}_{q}^{*}$ given by (1.13) and $z \in H_{q}$. If $a=0$, then $E_{\chi, \operatorname{SL}\left(2, \mathbb{F}_{p}\right)}(z)=p\left(p^{2}-1\right)$. If

$$
a \in\left\{\frac{p-1}{2}, \frac{2(p-1)}{2}, \frac{3(p-1)}{2}, \frac{4(p-1)}{2}, \ldots, \frac{m(p-1)}{2}\right\},
$$

where $m / 2=(q-1) /(p-1)-1$, then we have the Fourier expansion

$$
\begin{aligned}
\frac{1}{p(p-1)} E_{\chi, \operatorname{SL}\left(2, \mathbb{F}_{p}\right)}(z)= & \chi(y)+\frac{p}{q} \frac{\Gamma\left(\lambda, \Psi_{1}\right) \Gamma\left(\lambda \chi, \Psi_{1}\right)}{\Gamma\left(\chi, \Psi_{1}\right)} \chi^{-1}(-\delta y) \lambda(-\delta) \\
& +\frac{p}{q} \frac{\Gamma\left(\lambda, \Psi_{1}\right)}{\Gamma\left(\chi, \Psi_{1}\right)} \chi(y) \sum_{\substack{0 \neq b \in \mathbb{F}_{q} \\
\operatorname{Tr}(b)=0}}(\chi \lambda)(b) K_{\Psi_{b}}\left(\lambda \chi \mid-\delta y^{2},-\frac{1}{4}\right) \psi_{b}(-x) .
\end{aligned}
$$

If $a \notin\{0,(p-1) / 2,2(p-1) / 2,3(p-1) / 2,4(p-1) / 2, \ldots, m(p-1) / 2\}$, then $E_{\chi, \operatorname{SL}\left(2, \mathbb{F}_{p}\right)}(z)=0$.

If $q \neq p^{2}$ and $a \in\{0,(p-1) / 2,2(p-1) / 2,3(p-1) / 2,4(p-1) / 2, \ldots, m(p-1) / 2\}$, where $m / 2=(q-1) /(p-1)-1$, then $E_{\chi, S L\left(2, \mathbb{F}_{p}\right)}(z)$ is not the zero function.

Similar results hold for $E_{\chi, \mathrm{GL}\left(2, \mathbb{F}_{p}\right)}(z)$. Let $\chi=\chi_{a}$ be the multiplicative character of $\mathbb{F}_{q}^{*}$ given by (1.13) and $z \in H_{q}$. If $a=0$, then $E_{\chi, \mathrm{GL}\left(2, \mathbb{F}_{p}\right)}(z)=p(p-1)\left(p^{2}-1\right)$. If

$$
a \in\{p-1,2(p-1), \ldots, m(p-1)\}
$$

where $m=(q-1) /(p-1)-1$, then

$$
\begin{aligned}
\frac{1}{p(p-1)^{2}} E_{\chi, \mathrm{GL}\left(2, \mathbb{F}_{p}\right)}(z)= & \chi(y)+\frac{p}{q} \frac{\Gamma\left(\lambda, \Psi_{1}\right) \Gamma\left(\lambda \chi, \Psi_{1}\right)}{\Gamma\left(\chi, \Psi_{1}\right)} \chi^{-1}(-\delta y) \lambda(-\delta) \\
& +\frac{p}{q} \frac{\Gamma\left(\lambda, \Psi_{1}\right)}{\Gamma\left(\chi, \Psi_{1}\right)} \chi(y) \sum_{\substack{0 \neq b \in \mathbb{F}_{q} \\
\operatorname{Tr}(b)=0}}(\chi \lambda)(b) K_{\Psi_{b}}\left(\lambda \chi \mid-\delta y^{2},-\frac{1}{4}\right) \psi_{b}(-x) .
\end{aligned}
$$

If $a \notin\{0, p-1,2(p-1), \ldots, m(p-1)\}$, then $E_{\chi, \mathrm{GL}\left(2, \mathbb{F}_{p}\right)}(z)=0$.

If $q \neq p^{2}$ and $a \in\{0, p-1,2(p-1), \ldots, m(p-1)\}$, where $m=(q-1) /(p-1)-1$, then $E_{\chi, \mathrm{GL}\left(2, \mathbb{F}_{p}\right)}(z)$ is not the zero function.

In order to compute some values of these Eisenstein series, we need to think about fundamental domains. Here we will only consider $q=p^{2}$ for $p=3,5$. The fundamental domains in Examples 2.13 and 2.14 and the Eisenstein series in Tables 2.1 and 2.2 were calculated using Mathematica. 


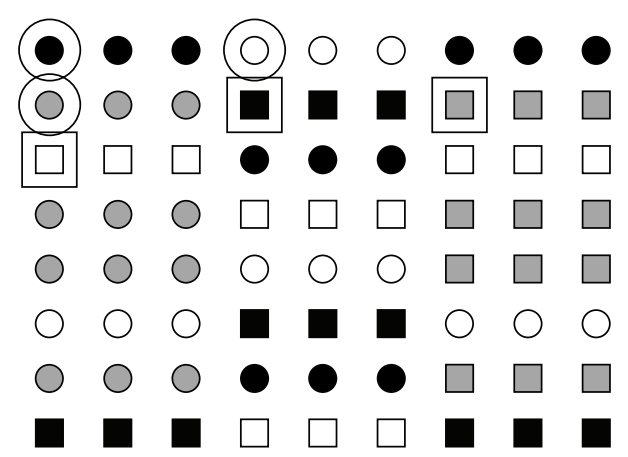

Figure 2.2. The fundamental domain for $\operatorname{SL}\left(2, \mathbb{F}_{3} \backslash H_{9}\right)$ is given by the 6 points which are either circled or boxed.

Example $2.13\left(\mathrm{SL}\left(2, \mathbb{F}_{3}\right) \backslash H_{9}\right)$. Write $x$ for a root of $x^{2}+1=0$ and $\mathbb{F}_{9}=\{a+b x \mid a, b \in \mathbb{F}\}$. Then let $\delta=1+x$, and suppose $y$ is a root of the equation $y^{2}=\delta$. Then, $H_{9}=\{a+$ $\left.b y \mid a, b \in \mathbb{F}_{9}, b \neq 0\right\}$. The elements of $\mathbb{F}_{9}$ are given the ordering $\{1+2 x, 2+2 x, 2 x, 2,0,1$, $x, 1+x, 2+x\}$. Each SL(2, $\left.\mathbb{F}_{3}\right)$-orbit in Figure 2.2 has a different shape and level of shading. The elements of the fundamental domain have been circled (or boxed). Reading from left to right increases $\operatorname{Re}(z)$, and reading from bottom to top increases $\operatorname{Im}(z)$. More explicitly, a fundamental domain for $\operatorname{SL}\left(2, \mathbb{F}_{3}\right) \backslash H_{9}$ is the set

$$
\begin{aligned}
& \{1+2 x+(2+x) y, 2+(2+x) y, 1+2 x+(1+x) y, \\
& 2+(1+x) y, x+(1+x) y, 1+2 x+x y\} .
\end{aligned}
$$

Example $2.14\left(\mathrm{SL}\left(2, \mathbb{F}_{5}\right) \backslash H_{25}\right)$. Let $x$ be a root of $x^{2}+2=0$ and $\mathbb{F}_{25}=\left\{a+b x \mid a, b \in \mathbb{F}_{5}\right\}$. Suppose $\delta=1+x$, and $y$ satisfies $y^{2}=\delta$. Then we have $H_{25}=\left\{a+b y \mid a, b \in \mathbb{F}_{25}, b \neq 0\right\}$. It can be shown that a fundamental domain for $\operatorname{SL}\left(2, \mathbb{F}_{5}\right) \backslash H_{25}$ is given by the 10 points

$$
\begin{aligned}
& \{1+3 x+(4+2 x) y, 1+4 x+(4+2 x) y, 3+(4+2 x) y, 2 x+(4+2 x) y, 3+(3+2 x) y, \\
& 1+4 x+(2+2 x) y, x+(2+2 x) y, 1+4 x+(1+2 x) y, 3+(1+2 x) y, 1+4 x+(4+x) y\} .
\end{aligned}
$$

We will write $A, B, C, D, E, F, G, H, I, J$ for these 10 points to save space in Table 2.2.

More general results on fundamental domains can be found in [13].

Finally, we compute values of $E_{\chi, \mathrm{SL}\left(2, \mathbb{F}_{p}\right)}(z)$ for the preceding examples. One can compute $E_{\chi, \mathrm{GL}\left(2, \mathbb{F}_{p}\right)}(z)$ for the same values of $p$ and $q$ using formula (2.29).

Table 2.1 gives the values of $E_{\chi, \operatorname{SL}\left(2, \mathbb{F}_{3}\right)}$ on $\operatorname{SL}\left(2, \mathbb{F}_{3}\right) \backslash H_{9}$ for $\chi=\chi_{a}, 0 \leq a \leq 7$. Table 2.2 gives the values of $E_{\chi, \mathrm{SL}\left(2, \mathbb{F}_{5}\right)}$ on $\mathrm{SL}\left(2, \mathbb{F}_{5}\right) \backslash H_{25}$ for $\chi=\chi_{a}, 0 \leq a \leq 23$. It follows from our computations that at least for these special cases the Eisenstein series on $H_{p^{2}}$ for $\operatorname{SL}\left(2, \mathbb{F}_{p}\right)$ do not vanish. 
14 Fourier expansions of Eisenstein series

Table 2.1. Values of $E_{\chi, \mathrm{SL}\left(2, \mathbb{F}_{3}\right)}$ on $\operatorname{SL}\left(2, \mathbb{F}_{3}\right) \backslash H_{9}$ for $\chi=\chi_{a}, 0 \leq a \leq 7$.

\begin{tabular}{|c|c|c|}
\hline$z$ & $E_{\chi_{0}, S L\left(2, \mathbb{F}_{3}\right)}$ & $E_{\chi_{1}, \operatorname{SL}\left(2, \mathbb{F}_{3}\right)}$ \\
\hline $1+2 x+(2+x) y$ & 24 & $-6 i+(3-9 i) \sqrt{2}$ \\
\hline $2+(2+x) y$ & 24 & $(-6+12 i)+(3-3 i) \sqrt{2}$ \\
\hline $1+2 x+(1+x) y$ & 24 & 0 \\
\hline $2+(1+x) y$ & 24 & $6 i-(3-9 i) \sqrt{2}$ \\
\hline$x+(1+x) y$ & 24 & 0 \\
\hline $1+2 x+x y$ & 24 & $(6-12 i)-(3-3 i) \sqrt{2}$ \\
\hline$z$ & $E_{\chi_{2}, \operatorname{SL}\left(2, \mathbb{F}_{3}\right)}$ & $E_{\chi_{3}, \operatorname{SL}\left(2, \mathbb{F}_{3}\right)}$ \\
\hline $1+2 x+(2+x) y$ & $-6-6 i$ & $6 i-(3+9 i) \sqrt{2}$ \\
\hline $2+(2+x) y$ & $-6-6 i$ & $-6-12 i-(3+3 i) \sqrt{2}$ \\
\hline $1+2 x+(1+x) y$ & $12+12 i$ & 0 \\
\hline $2+(1+x) y$ & $-6-6 i$ & $-6 i+(3+9 i) \sqrt{2}$ \\
\hline$x+(1+x) y$ & $12+12 i$ & 0 \\
\hline $1+2 x+x y$ & $-6-6 i$ & $6+12 i+(3+3 i) \sqrt{2}$ \\
\hline$z$ & $E_{\chi_{4}, \operatorname{SL}\left(2, \mathbb{F}_{3}\right)}$ & $E_{\chi_{5}, \mathrm{SL}\left(2, \mathbb{F}_{3}\right)}$ \\
\hline $1+2 x+(2+x) y$ & -12 & $-6 i-(3-9 i) \sqrt{2}$ \\
\hline $2+(2+x) y$ & 12 & $-6+12 i-(3-3 i) \sqrt{2}$ \\
\hline $1+2 x+(1+x) y$ & 0 & 0 \\
\hline $2+(1+x) y$ & -12 & $6 i+(3-9 i) \sqrt{2}$ \\
\hline$x+(1+x) y$ & 0 & 0 \\
\hline $1+2 x+x y$ & 12 & $6-12 i+(3-3 i) \sqrt{2}$ \\
\hline$z$ & $E_{\chi_{6}, \operatorname{SL}\left(2, \mathbb{F}_{3}\right)}$ & $E_{\chi_{7}, \mathrm{SL}\left(2, \mathbb{F}_{3}\right)}$ \\
\hline $1+2 x+(2+x) y$ & $-6+6 i$ & $6 i+(3+9 i) \sqrt{2}$ \\
\hline $2+(2+x) y$ & $-6+6 i$ & $-6-12 i+(3+3 i) \sqrt{2}$ \\
\hline $1+2 x+(1+x) y$ & $12-12 i$ & 0 \\
\hline $2+(1+x) y$ & $-6+6 i$ & $-6 i-(3+9 i) \sqrt{2}$ \\
\hline$x+(1+x) y$ & $12-12 i$ & 0 \\
\hline $1+2 x+x y$ & $-6+6 i$ & $6+12 i-(3+3 i) \sqrt{2}$ \\
\hline
\end{tabular}


Table 2.2. Values of $E_{\chi, \Gamma}$ on $\Gamma \backslash H_{25}$ for $\chi=\chi_{a}, 0 \leq a \leq 23, \Gamma=\operatorname{SL}\left(2, \mathbb{F}_{5}\right)$.

\begin{tabular}{|c|c|c|c|}
\hline$z$ & $E_{\chi_{0}, \operatorname{SL}\left(2, \mathbb{F}_{5}\right)}(z)$ & $E_{\chi_{2}, \operatorname{SL}\left(2, \mathbb{F}_{5}\right)}(z)$ & $E_{\chi_{4}, \mathrm{SL}\left(2, \mathbb{F}_{5}\right)}(z)$ \\
\hline$A$ & 120 & 0 & $20+20 i \sqrt{3}$ \\
\hline$B$ & 120 & $-30-10 i-(10-30 i) \sqrt{3}$ & $20+20 i \sqrt{3}$ \\
\hline C & 120 & $30-40 i+(20-10 i) \sqrt{3}$ & $-30-30 i \sqrt{3}$ \\
\hline$D$ & 120 & 0 & $20+20 i \sqrt{3}$ \\
\hline E & 120 & $-50 i+(10+20 i) \sqrt{3}$ & $-30-30 i \sqrt{3}$ \\
\hline$F$ & 120 & 0 & $20+20 i \sqrt{3}$ \\
\hline$G$ & 120 & 0 & $20+20 i \sqrt{3}$ \\
\hline$H$ & 120 & $50 i-(10+20 i) \sqrt{3}$ & $-30-30 i \sqrt{3}$ \\
\hline$I$ & 120 & $30+10 i+(10-30 i) \sqrt{3}$ & $20+20 i \sqrt{3}$ \\
\hline$J$ & 120 & $-30+40 i-(20-10 i) \sqrt{3}$ & $-30-30 i \sqrt{3}$ \\
\hline$z$ & $E_{\chi_{6}, \operatorname{SL}\left(2, \mathbb{F}_{5}\right)}(z)$ & $E_{\chi_{8}, \mathrm{SL}\left(2, \mathbb{F}_{5}\right)}(z)$ & $E_{\chi_{10}, \operatorname{SL}\left(2, \mathbb{F}_{5}\right)}(z)$ \\
\hline$A$ & 0 & $-60-20 i \sqrt{3}$ & 0 \\
\hline$B$ & $-60-20 i$ & 0 & $-30-10 i+(10-30 i) \sqrt{3}$ \\
\hline C & $-60-20 i$ & $-30-10 i \sqrt{3}$ & $30-40 i-(20-10 i) \sqrt{3}$ \\
\hline$D$ & 0 & $-60-20 i \sqrt{3}$ & 0 \\
\hline$E$ & $60+20 i$ & $30+10 i \sqrt{3}$ & $-50 i-(10+20 i) \sqrt{3}$ \\
\hline$F$ & 0 & $60+20 i \sqrt{3}$ & 0 \\
\hline G & 0 & $60+20 i \sqrt{3}$ & 0 \\
\hline$H$ & $-60-20 i$ & $30+10 i \sqrt{3}$ & $50 i+(10+20 i) \sqrt{3}$ \\
\hline$I$ & $60+20 i$ & 0 & $30+10 i-(10-30 i) \sqrt{3}$ \\
\hline$J$ & $60+20 i$ & $-30-10 i \sqrt{3}$ & $-30+40 i+(20-10 i) \sqrt{3}$ \\
\hline$z$ & $E_{\chi_{12}, \operatorname{SL}\left(2, \mathbb{F}_{5}\right)}(z)$ & $E_{\chi_{14}, \operatorname{SL}\left(2, \mathbb{F}_{5}\right)}(z)$ & $E_{\chi_{16}, \operatorname{SL}\left(2, \mathbb{F}_{5}\right)}(z)$ \\
\hline$A$ & -40 & 0 & $-60+20 i \sqrt{3}$ \\
\hline$B$ & 80 & $-30+10 i+(10+30 i) \sqrt{3}$ & 0 \\
\hline C & 0 & $30+40 i-(20+10 i) \sqrt{3}$ & $-30+10 i \sqrt{3}$ \\
\hline$D$ & -40 & 0 & $-60+20 i \sqrt{3}$ \\
\hline$E$ & 0 & $50 i-(10-20 i) \sqrt{3}$ & $30-10 i \sqrt{3}$ \\
\hline$F$ & -40 & 0 & $60-20 i \sqrt{3}$ \\
\hline$G$ & -40 & 0 & $60-20 i \sqrt{3}$ \\
\hline$H$ & 0 & $-50 i+(10-20 i) \sqrt{3}$ & $30-10 i \sqrt{3}$ \\
\hline$I$ & 80 & $30-10 i-(10+30 i) \sqrt{3}$ & 0 \\
\hline$J$ & 0 & $-30-40 i+(20+10 i) \sqrt{3}$ & $-30+10 i \sqrt{3}$ \\
\hline
\end{tabular}


Table 2.2. Continued.

\begin{tabular}{c|ccc}
\hline$z$ & $E_{\chi_{18}, \mathrm{SL}\left(2, \mathbb{F}_{5}\right)}(z)$ & $E_{\chi_{20}, \mathrm{SL}\left(2, \mathbb{F}_{5}\right)}(z)$ & $E_{\chi_{22}, \mathrm{SL}\left(2, \mathbb{F}_{5}\right)}(z)$ \\
\hline$A$ & 0 & $20-20 i \sqrt{3}$ & 0 \\
$B$ & $-60+20 i$ & $20-20 i \sqrt{3}$ & $-30+10 i-(10+30 i) \sqrt{3}$ \\
$C$ & $-60+20 i$ & $-30+30 i \sqrt{3}$ & $30+40 i+(20+10 i) \sqrt{3}$ \\
$D$ & 0 & $20-20 i \sqrt{3}$ & 0 \\
$E$ & $60-20 i$ & $-30+30 i \sqrt{3}$ & $50 i+(10-20 i) \sqrt{3}$ \\
$F$ & 0 & $20-20 i \sqrt{3}$ & 0 \\
$G$ & 0 & $20-20 i \sqrt{3}$ & $-50 i-(10-20 i) \sqrt{3}$ \\
$H$ & $-60+20 i$ & $-30+30 i \sqrt{3}$ & $30-10 i-(10+30 i) \sqrt{3}$ \\
$I$ & $60-20 i$ & $20-20 i \sqrt{3}$ & $-30-40 i-(20+10 i) \sqrt{3}$ \\
$J$ & $60-20 i$ & $-30+30 i \sqrt{3}$ & \\
\hline
\end{tabular}

\section{References}

[1] J. Angel, Finite upper half planes over finite fields, Finite Fields and Their Applications 2 (1996), no. $1,62-86$.

[2] J. Angel, N. Celniker, S. Poulos, A. Terras, C. Trimble, and E. Velasquez, Special functions on finite upper half planes, Hypergeometric Functions on Domains of Positivity, Jack Polynomials, and Applications (Tampa, FL, 1991), Contemp. Math., vol. 138, American Mathematical Society, Rhode Island, 1992, pp. 1-26.

[3] J. Angel and R. Evans, Diameters of finite upper half plane graphs, Journal of Graph Theory 23 (1996), no. 2, 129-137.

[4] E. Bannai and T. Ito, Algebraic Combinatorics I: Association Schemes, The Benjamin/Cummings, California, 1984.

[5] P. T. Bateman and E. Grosswald, On Epstein's zeta function, Acta Arithmetica 9 (1964), 365-373.

[6] N. Celniker, S. Poulos, A. Terras, C. Trimble, and E. Velasquez, Is there life on finite upper half planes?, A Tribute to Emil Grosswald: Number Theory and Related Analysis, Contemp. Math., vol. 143, American Mathematical Society, Rhode Island, 1993, pp. 65-88.

[7] C.-L. Chai and W.-C. W. Li, Character sums, automorphic forms, equidistribution, and Ramanujan graphs. II: eigenvalues of Terras graphs, Forum Mathematicum 16 (2004), no. 5, 631-661.

[8] R. Evans, Spherical functions for finite upper half planes with characteristic 2, Finite Fields and Their Applications 1 (1995), no. 3, 376-394.

[9] D. M. Hejhal, The Selberg Trace Formula for PSL $(2, \mathbb{R})$. Vol. I, Lecture Notes in Mathematics, vol. 548, Springer, New York, 1976.

[10] K. F. Ireland and M. I. Rosen, A Classical Introduction to Modern Number Theory, Graduate Texts in Mathematics, vol. 84, Springer, New York, 1982.

[11] A. Krieg, Hecke algebras, Memoirs of the American Mathematical Society 87 (1990), no. 435, $\mathrm{x}+158$.

[12] P. Sarnak, Kloosterman, quadratic forms and modular forms, Nieuw Archief voor Wiskunde. Vijfde Serie (5)1 (2000), no. 4, 385-389.

[13] A. Shaheen, Finite planes and finite upper half planes: their geometry, a trace formula, modular forms and eisenstein series, Ph.D. thesis, University of California San Diego, California, 2005. 
[14] J. H. Silverman, Advanced Topics in the Arithmetic of Elliptic Curves, Graduate Texts in Mathematics, vol. 151, Springer, New York, 1994.

[15] J. Soto-Andrade and J. Vargas, Twisted spherical functions on the finite Poincaré upper half-plane, Journal of Algebra 248 (2002), no. 2, 724-746.

[16] A. Terras, Bessel series expansions of the Epstein zeta function and the functional equation, Transactions of the American Mathematical Society 183 (1973), 477-486.

[17] _ Harmonic Analysis on Symmetric Spaces and Applications. Vol.I, Springer, New York, 1985.

[18] _ Fourier Analysis on Finite Groups and Applications, London Mathematical Society Student Texts, vol. 43, Cambridge University Press, Cambridge, 1999.

[19] P. D. Tiu and D. I. Wallace, Norm quadratic-residue codes, IEEE Transactions on Information Theory 40 (1994), no. 3, 946-949.

Anthony Shaheen: Department of Mathematics, California State University, Los Angeles, CA 90032-8204, USA

E-mail address: ashahee@exchange.calstatela.edu

Audrey Terras: Department of Mathematics, University of California, San Diego, La Jolla, CA 92093-0112, USA

E-mail address: aterras@math.ucsd.edu 


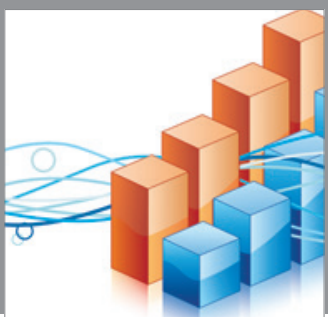

Advances in

Operations Research

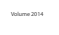

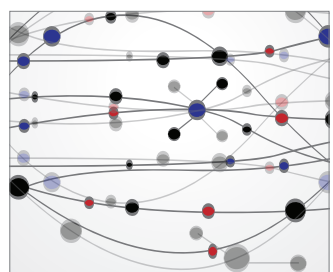

\section{The Scientific} World Journal
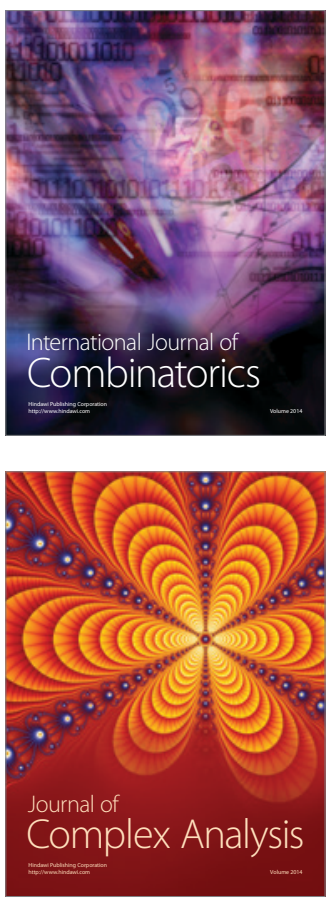

International Journal of

Mathematics and

Mathematical

Sciences
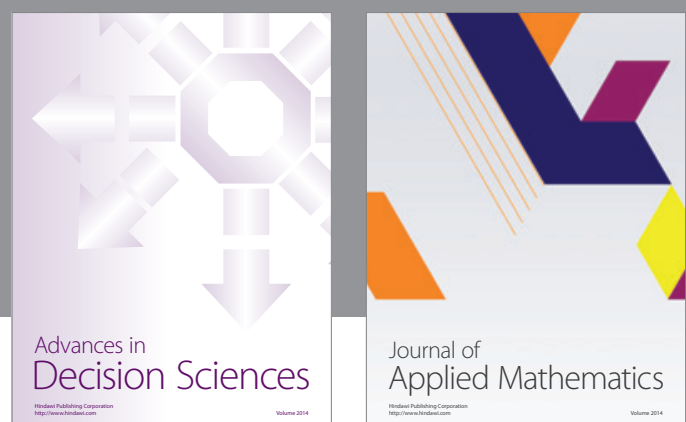

Journal of

Applied Mathematics
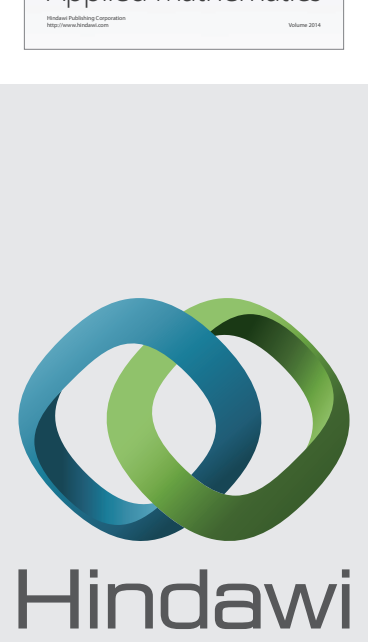

Submit your manuscripts at http://www.hindawi.com
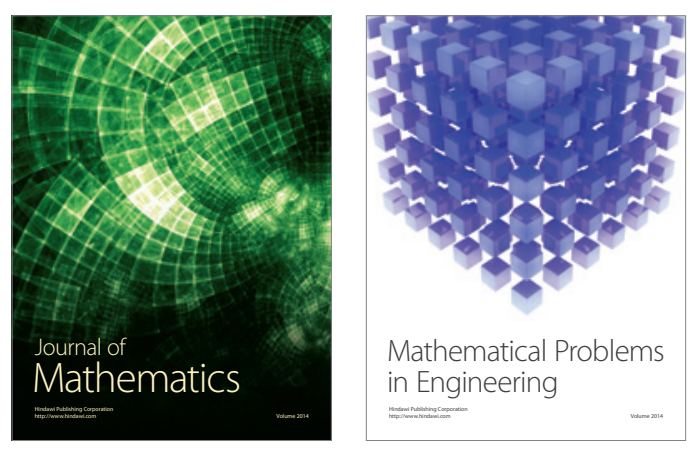

Mathematical Problems in Engineering
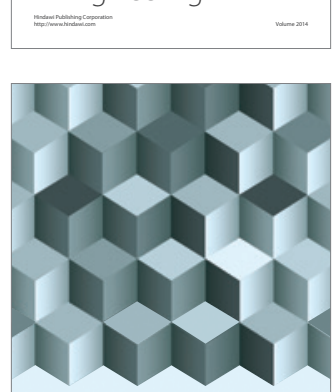

Journal of

Function Spaces
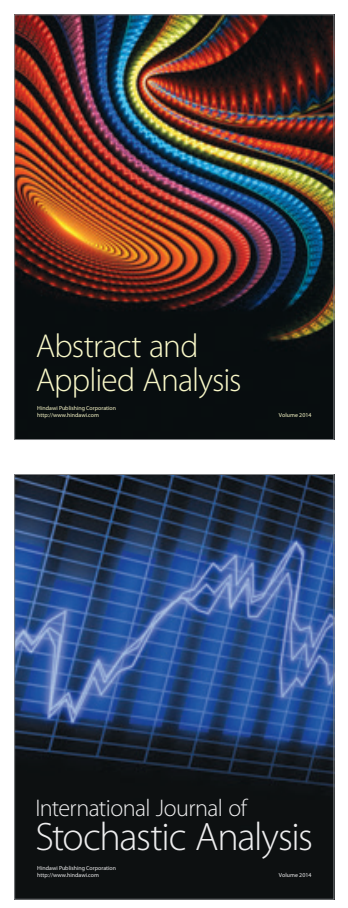

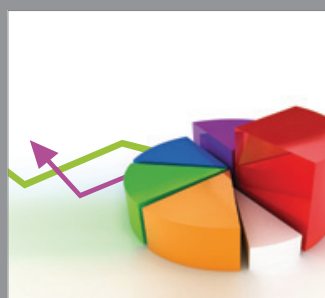

ournal of

Probability and Statistics

Promensencen
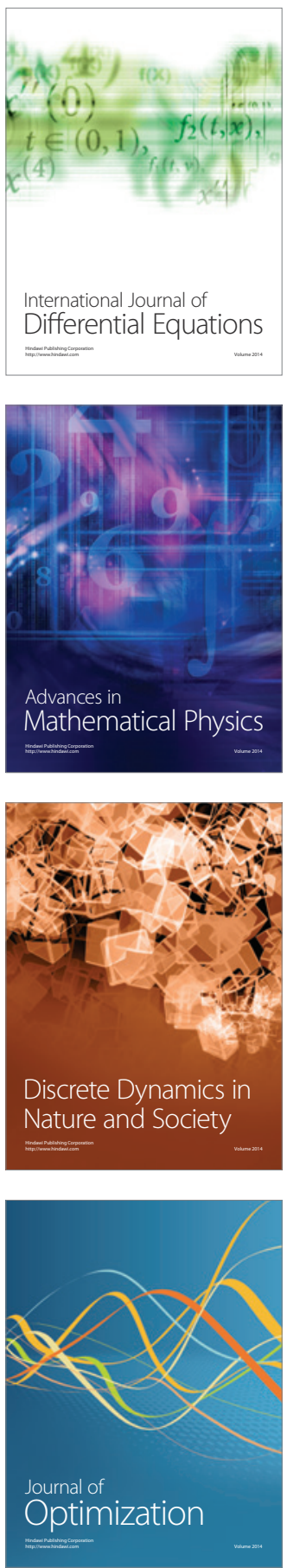